\title{
Cinémas
}

Revue d'études cinématographiques

Journal of Film Studies

\section{Richard Bégin, Myriam Dussault et Emmanuelle Dyotte (dir.), La circulation des images. Médiation des cultures, Paris, L'Harmattan, 2006, 214 p.}

\section{Philippe Théophanidis}

Volume 17, numéro 2-3, printemps 2007

La théorie du cinéma, enfin en crise

URI : https://id.erudit.org/iderudit/016757ar

DOI : https://doi.org/10.7202/016757ar

Aller au sommaire du numéro

Éditeur(s)

Cinémas

ISSN

1181-6945 (imprimé)

1705-6500 (numérique)

Découvrir la revue

Citer ce compte rendu

Théophanidis, P. (2007). Compte rendu de [Richard Bégin, Myriam Dussault et Emmanuelle Dyotte (dir.), La circulation des images. Médiation des cultures, Paris, L’Harmattan, 2006, 214 p.] Cinémas, 17(2-3), 235-248.

https://doi.org/10.7202/016757ar d'utilisation que vous pouvez consulter en ligne. 
Richard Bégin, Myriam Dussault et Emmanuelle Dyotte (dir.), La circulation des images. Médiation des cultures, Paris, L'Harmattan, 2006, 214 p.

Depuis plusieurs années déjà, il est de pratique courante pour les sciences humaines de questionner l'efficace des repères que nous fabriquons et qui informent en retour nos manières d'être. Certains ont pu suggérer que ce discours s'élabore précisément à partir d'une transformation radicale des moyens par lesquels nous parvenons à mettre en forme nos expériences. Aussi, s'il peut sembler excessif d'évoquer la fin générale de la représentation, il demeure néanmoins pertinent d'interroger les variations que peuvent manifester les divers régimes représentatifs auxquels nous sommes exposés. En ce sens, le collectif publié récemment sous le titre La circulation des images ${ }^{1}$ offre un double intérêt. D'une part, la plupart des textes qui le constituent donnent à penser des objets d'analyse dont le fonctionnement "problématise» le concept de représentation. D'autre part, cette publication est elle-même l'expression, lisible, de la forme et de l'orientation que peuvent adopter aujourd'hui certains discours institutionnels préoccupés par cette problématique.

À partir du constat de la prolifération généralisée des "images ${ }^{2}$ » et du rôle important qu'elles jouent dans l'organisation de nos expériences, les quatorze auteurs de ce collectif proposent l'analyse de divers objets, œuvres ou phénomènes. Malgré la diversité des perspectives théoriques mobilisées, la plupart des textes partagent un certain nombre de points communs, exprimés tantôt explicitement comme postulats théoriques, tantôt comme cadre méthodologique, tantôt comme conclusion implicite.

Toutes ces analyses tendent en effet à aborder l'image en questionnant l'idée générale d'une représentation dont le rôle consisterait essentiellement à rendre compte d'un référent 
normatif (compris comme «essentiel» ou "authentique»). Une volonté de comprendre autrement les possibilités créatrices liées à la "circulation des images" semble à l'œuvre tant dans les objets abordés que dans la structure même des études qu'elles motivent. Dès lors, l'image ne s'étudie plus comme une unité autonome, mais bien comme l'émergence perceptible, temporairement stabilisée, d'un processus relationnel complexe rassemblant matériaux d'expression, moyens de production, gestes artistiques, regards spectatoriels, discours théoriques, etc. ${ }^{3}$. Dans cette perspective, l'œuvre et la communauté sont coproduites par les rapports qu'elles entretiennent. Il n'est donc pas étonnant que la "circulation des images" soit également associée à des effets de réel. Ce sont en définitive ces effets qui semblent intéresser, de façon générale, les auteurs réunis dans ce collectif. Je propose ici un survol rapide de chacun de ces textes en respectant l'ordre de présentation de l'édition qui les rassemble.

Olivier Asselin propose en premier lieu un essai sur le phénomène d'autoreprésentation associé à la multiplication des sites amateurs offrant un accès à des webcams personnelles. L'auteur relève rapidement la problématique essentielle associée à ce type de spectacle. En effet, de manière générale, "ces sites ne donnent rien à voir" et la vie de ceux qui s'y donnent en représentation semble "sans événement heureux, ni malheureux, sans passé et sans avenir» (p. 11). Néanmoins, ces sites connaissent une popularité certaine. Asselin développe son essai sous la forme d'un triptyque interrogeant la nature des rapports ainsi médiatisés entre regardant et regardé. Un premier volet esquisse une critique de la spectacularisation des rapports sociaux. Un deuxième aborde rapidement le rôle du dispositif panoptique pour une société dite, avec Michel Foucault, "disciplinaire». Un dernier volet, enfin, s'attarde sur le rôle des pulsions de désir et d'angoisse dans la mise en forme des relations entre voyeur et exhibitionniste par l'entremise de cet "ombilic technologique» (p. 18). L'argument principal de cet essai vise à souligner le développement d'une curieuse intimité qui relèverait moins d'une communication (comprise dans le sens restreint d'un processus d'échange) que d'une communion (p. 19). Asselin évoque ainsi une préoccupation théorique déjà 
bien connue et que l'on retrouvera par ailleurs dans plusieurs autres essais de ce collectif: la médiatisation des expériences vécues tend à informer des rapports dans lequel le plus intime n'est pas nécessairement le plus proche ${ }^{4}$.

L'intérêt du texte de Richard Bégin sur le rôle des ruines urbaines dans les films de fiction tient peut-être précisément à ce qu'il cerne un dispositif médiatique capable de présenter l'irreprésentable d'un temps révolu, de rendre proche un infiniment lointain. En l'absence de modèle, ou de référent, il ne peut y avoir de représentation. L'«irreprésentabilité» de cette absence peut néanmoins être exprimée, c'est-à-dire qu'elle peut être présentée à l'expérience d'un sujet sensible. L'évocation des restes du World Trade Center dans une séquence du film 25th Hour de Spike Lee (2002) est ici associée à une force expressive suffisante pour "ouvr[ir] l'histoire racontée sur une puissance de l'indicible» (p. 30). Le spectateur du film, saisi par la coïncidence entre "les affects du réel et ceux de la fiction" (p. 25), expérimente donc l'image de ces ruines comme un «sentiment de survivance» (p. 27). Un tel sentiment est imputable plus à la présentation d'un "temps pur " jaillissant de la fiction (littéralement: qui s'ex-prime), qu'à une simple relation référentielle avec l'événement historique transcendant l'œuvre de fiction.

La contribution de Baptiste Campion et Benoît Lambo constitue une nouvelle tentative afin de cerner, à partir d'une approche cognitiviste, les spécificités opérationnelles des images de synthèse offertes par les jeux vidéo. Le régime de représentation de ces «simulations" - dont la performance produit en quelque sorte le référent et se substitue ainsi à «la réalité» (p. 41 et 43) - est qualifié, avec Jean Baudrillard, d' $\mathrm{d}^{\text {hyperréel }}{ }^{5}{ }$. Les auteurs visent ainsi "à appréhender une approche de l'image reposant non plus sur une approche signifiant-signifié, mais bien sur une relation complexe entre perception, interprétation et modèles mentaux" (p. 51). Notons, toutefois, que ce plaidoyer en faveur "d'une prise en compte élargie" de la dimension cognitive de la "nature représentationnelle de l'image" (p. 52) semble paradoxalement opérer à partir d'une réduction - excessive, par moment - des concepts mobilisés (notamment ceux de virtualité et de simulation). Cette contribution a 
le mérite, d'une part, de susciter un questionnement sur le rapport entre les dimensions cognitive et sensible de l'expérience que nous faisons des images et, d'autre part, de réaffirmer le potentiel créatif - non pas simplement représentatif — de ces dernières.

À partir des tableaux vivants du photographe Édouard Levé et du chorégraphe Xavier Le Roy, Michelle Debat analyse précisément, avec rigueur et inventivité, le fonctionnement de l'image comme "mode de construction de relations" (p. 55) capable de "destitu[er] la représentation de la simple imitation» (p. 57). Le corps des sujets présentés est ici appréhendé moins comme la finalité du processus créatif que comme moyen produisant des «effets de réel» (p. 61, 63 et 68). Le travail que mène Debat sur la nature processuelle des relations dont sont constituées nos expériences rappelle certains des motifs avancés par William James au début du siècle dernier en faveur du développement d'un "empirisme radical ${ }^{6} »$. Ce faisant, l'auteure est l'une des rares à soulever l'enjeu éthique associé à la remise en question du régime de la représentation: "N'est-on pas d'ailleurs socialement et éthiquement dans une hiérarchie affirmée du relationnel sur le représenté: le premier usant du changement des manières d'être, le second privilégiant la stabilité des états des choses?» (p. 73). Ce constat est notamment rendu possible par la prise en compte — et Debat est la seule à le noter explicitement - de la double portée du concept de représentation: à la fois réflexive et transitive, éthique et politique (p. 68).

À l'instar de Campio et Lambo (remarques sur le «selfconsciousness» de l'image de synthèse) et de Debat (propos sur la présentation par l'œuvre chorégraphique de ses propres moyens de production), Emmanuelle Dyotte s'attarde sur la capacité qu'ont les œuvres performatives de l'artiste américaine Vanessa Beecroft de présenter les conditions mêmes qui rendent possible leur représentation (p. 76). Les expositions de Beecroft ${ }^{7}$, mettant en scène des séries de corps uniformes de femmes et d'hommes nus ou accessoirement vêtus, problématisent en effet leur consommation sous forme d'identité fétiche. Comme Debat, Dyotte appréhende le corps dans sa matérialité médiatique. Ainsi, pour Beecroft, «le médium corporel est toujours 
déjà entièrement investi par tout un ensemble de circonstances par rapport auxquelles il se construit comme un parmi d'autres et, également, se distingue» (p. 87). Aussi, si la multitude des corps exposés, figés en des poses artificielles, permet bien «la possibilité de la relation à travers l'identité collective», elle parvient néanmoins à "déplacer le regard fétichiste en rendant la transcendance impossible» (p. 85). Cette perspective permet à Dyotte d'attribuer une portée politique et critique à ces performances en suggérant qu'elles parviennent à opérationnaliser une résistance multiple aux "modes de vie normalisateurs" dont nous saturerait l'«Empire» - cette entité conceptuelle popularisée par les travaux de Michael Hardt et Antonio Negri (p. 83).

La remise en question d'une idéologie de l'unité ${ }^{8}$ à laquelle participe aujourd'hui cette "pensée du multiple» trouve une autre application dans l'excellent essai qu'offre André Habib. Ce dernier porte non pas, comme dans le texte de Richard Bégin, sur l'image de la ruine au cinéma, mais bien sur l'image ruinée $d u$ médium cinématographique lui-même. À partir du film Lyrisch Nitraat (1991) de Peter Delpeut, réalisé intégralement avec des archives cinématographiques du début du siècle, Habib propose l'analyse sentie d'une modalité de représentation particulière: celle de la «revenance» d'une unité expressive fragmentée (p. 100). Les archives assemblées par Delpeut sont en effet matériellement abîmées, ruinées. Leur présentation a le pouvoir de donner à sentir une présence inactuelle: celle d'un "temps visible qui se lit dans les vides et les marques qui fragmentent ou rongent la pellicule» (p. 100). Le film de Delpeut s'organise ainsi dans "l'horizon de cette perte», et son histoire "se présente comme fragment» (p. 101 et 103). La poétique de Lyrisch Nitraat n'est donc pas strictement redevable d'un référent, mais produite plutôt à partir d'un manque, «sa propre vérité esthétique» (p. 108). L'adroite démonstration d'Habib rejoint ici, d'une certaine manière, le postulat théorique avec lequel Debat ouvre son article lorsqu' elle avance "qu'il n'y a image, c'est-à-dire une image nommable, que lorsqu'il existe en négatif une part d'invisible qui fait lien pour que le sens puisse ouvrir à la parole» (p. 55).

L'idée selon laquelle l'image n'est plus nécessairement la fidèle et passive représentante d'un modèle normatif motive également 
l'analyse qu'offre Martin Legault de l'adaptation cinématographique du roman américain American Psycho (2000) réalisée par Mary Harron. L'étude de l'adaptation selon un « rapport de fidélité" y est en effet exposée comme un "confinement théorique» (p. 111-112). La perspective "intertextuelle», qu'il privilégie plutôt, attribue d'emblée une capacité créative à l'adaptation. Ainsi, le film apparaît comme une œuvre unique et originale engagée dans un espace d'intersection où circulent et dialoguent également les autres œuvres dont il s'inspire: notamment le roman écrit par Bret Easton Ellis, et une photographie particulière produite en 1966 par l'artiste new-yorkaise Cindy Sherman. S'appuyant sur cette "potentialité relationnelle" de l'image, Legault propose de concentrer son "regard théorique» sur le "discours du voyeurisme» actualisé par cette rencontre «intertextuelle»(p. 113). Dans sa série Untitled Film Stills, la photographe américaine ne se contente pas de reproduire passivement des clichés érotisant suggérés par les codes dominants de l'industrie cinématographique. Elle s'approprie ces codes et les exagère afin de créer ses propres "clichés". Son œuvre, en évoquant ainsi les conditions de possibilités même d'une consommation "scopophilique», parvient à en bousculer la dynamique (p. 119). Legault suppose qu'un processus similaire est à l'œuvre dans une séquence du film American Psycho où apparait une œuvre de Sherman: la cinéaste y met en scène la manière avec laquelle le protagoniste s'emploie à filmer ses ébats sexuels avec des prostituées. Autrement dit, lorsqu'une représentation offre un point de vue sur son propre fonctionnement ${ }^{9}$, elle empêche d'établir une relation efficace à la chose pour laquelle elle tient lieu et oblige ainsi à l'expérience de ses propres conditions de possibilités. La représentation acquiert alors un potentiel d'expression irréductible à ce qu'elle représente transitivement ${ }^{10}$. Michel Foucault (1966) offre une des plus brillantes illustrations de ce phénomène lorsqu'il s'attarde à l'interprétation, bien connue, du tableau Las Meninas de Velasquez ${ }^{11}$.

$\mathrm{Si}$, pour Guy Debord, la médiatisation par les images du rapport social qui unit les hommes est le signe d'une intensification "spectaculaire" du capital, pour Michel Maffesoli, elle est surtout l'occasion d'un rapprochement entre les hommes. Ce 
dernier propose moins, par son texte, l'analyse d'un objet spécifique qu'une réflexion plus générale sur le rôle que peut jouer l'«image partagée" pour la mise en forme d'une manière de vivre ensemble. L'enjeu visant à la "constitution d'un esprit commun ", ou "reliance imaginale», relève ici moins d'un rationalisme moralisateur que d'une "émotionnalité " associée à une "pensée organique» et holistique (p. 129-131). Il faut d'emblée signaler que la forme du texte de Maffesoli est également singulière, rappelant plus un pamphlet messianique qu'un texte d'analyse formaté selon la "doxa» institutionnelle. Un effet sans doute souhaité et, au demeurant, cohérent avec les thèses avancées par l'auteur. L’argumentaire développé est intéressant sous plusieurs aspects, notamment en ce qu'il conjugue et expose de manière emphatique certains des thèmes dispersés chez les autres participants: importance de la fonction de l'image pour la communication entre les sujets qui en font l'expérience, préoccupation marquée pour la dimension sensible ou affective associée aux divers régimes représentatifs, nécessité de déplacer l'attention de l'étude des termes à l'étude des relations qui les unissent et les produisent, etc. Néanmoins, l'enthousiasme senti que met Maffesoli lorsqu'il nous invite à "suivre le signe de piste du nomadisme tribal» (p. 137, c'est l'auteur qui souligne) ou encore à "transfigur[er] un quotidien dominé par la logique marchande» (p. 136) semble opérer au détriment d'une rigueur dans la démonstration. Par exemple, il lui semble nécessaire de justifier sa critique d'un certain conformisme rationaliste en mettant en scène une «intelligentsia moderne» — pure invention discursive - qu'il s'acharne à accabler de tous les maux. Il encourage encore à «ne plus se plier à une logique de la séparation" (p. 132), mais ne peut s'empêcher de distinguer entre moderne et postmoderne, entre ce qui est et ce qui devrait être. Cette critique prescriptive possède néanmoins l'indiscutable mérite d'appeler explicitement au débat d'idées.

Dans le magnifique essai qu'elle consacre au cinéaste chilien Raoul Ruiz, Marie-Hélène Mello analyse avec adresse le potentiel créatif de l'«image relationnelle» (p. 140) qui fait se rencontrer à la fois discours théoriques, écriture littéraire, peinture, mouvement théâtral et temporalité cinématographique. 
L'auteure suggère d'emblée que "le tableau vivant joue sur le simulacre, sur les limites de la représentation et sur les paradoxes de la présentation»(p. 140-141). Toutefois, contrairement à Campo et Lambio, Mello n'envisage pas ce simulacre dans la perspective de la différence de degré qu'il entretient avec un modèle normatif auquel il viendrait finalement se substituer. Le simulacre qu'elle donne à penser, avec Klossowski et Deleuze, est une puissance d'actualisation qualitativement - et radicalement - distincte de la fonction remplie par la notion de modèle. Le film L'hypothèse du tableau volé (1978) de Ruiz ne s'offre pas comme la représentation fabuleuse de tableaux réels: son événement réalise plutôt les conditions permettant la présentation d'une "fiction théorique»; il ne se substitue pas au réel: il produit un réel. Cette réalité, c’est celle de la tension temporelle induite par le tableau vivant et qui «rend la durée sensible» (p. 149). C'est celle encore du caractère éphémère de l'expérience cinématographique. Celle aussi, sans doute, du texte que Marie-Hélène Mello nous donne à lire.

Lucie Roy propose un long développement librement inspiré de l'idée générale selon laquelle un faisceau de relations est susceptible d'informer les termes qu'il unit. Ce faisant, sa démonstration vise à dégager les spécificités d'un régime d'expression cinématographique particulier à l'œuvre dans le cinéma de Pierre Perrault. Parce que l'«écriture» de ce dernier est "fondée sur la rencontre de l'un (le réalisateur) et de l'autre (les personnes qu'il a connues)", l'auteure propose - avec une perspicacité certaine - de la qualifier d' "expériencielle» (p. 153). L'analyse qui en résulte, par son mode d'expression et sa complexité, laisse songeur. Ainsi de ce passage exemplaire où il est avancé que la "forme du film», identifiée par l'auteure à "l'écriture filmique ${ }^{12}$ ", donne à lire les enquêtes que Perrault a menées "auprès de ceux qui vivent ", enquêtes ayant activement "modulé le travail symbolique du film» (p. 158). Ce processus de modulation est lui-même fonction des régimes de perception qu'exercent les sujets $d u$ film, les sujets qui font le film et les sujets qui regardent le film ${ }^{13}$. À la lumière de ce raisonnement, l'auteure suggère encore: 
Autrement dit, ce serait, par endroit et à titre d'exemple, la relation - qui s'offre à la perception spectatorielle dans ce cas-ci - établie par l'énonciateur ou l'observateur et l'intervieweur avec les observés ou les interviewés qui aurait - formellement — modulé le contenu du film dans la mesure où, je le souligne, c'est la relation qui le qualifie (p. 158).

Cela signifie-t-il que le film, au niveau de sa forme et de son contenu, est notamment conditionné par les fonctions perceptives qu'il mobilise? Est-ce que la forme et le contenu se coproduisent mutuellement? Quel régime causal serait impliqué par une telle simultanéité? Les flottements similaires ${ }^{14}$ qui accompagnent le déploiement de cette analyse n'empêchent toutefois pas de relever quelques idées intéressantes. La référence rapide au concept de "transduction", indirectement emprunté à la philosophie de Gilbert Simondon, soulève par exemple la problématique liée au processus d'individuation de l'œuvre représentative en regard des notions de «modèle» et de «modulation" mobilisées par les rapports entre la forme et la matière ${ }^{15}$.

Les rapports qu'entretiennent observateurs et observés sont également au centre de l'étude que Thérèse Saint-Gelais consacre au motif du regard. En s'inspirant des œuvres de Mary Cassatt, Dominique Blain, Louise Blouin et Geneviève Cadieux, l'auteure examine

[...] quelques regards qui [lui] apparaissent, tous autant qu'ils sont, des regards de diverses connivences, mais dont l'espace de relation fait preuve d'échanges sensibles très singuliers en ce qu'ils interrogent directement ou en marge un investissement envers l'Autre qui, n'étant pas exempt à la fois de réserve et d'empathie significative, s'engage expressément dans l'espace relationnel qu'il se réserve (p. 175).

Dans ses grandes lignes, le travail de Saint-Gelais rejoint cette idée selon laquelle les limites de la représentation surgissent au moment où celle-ci acquiert une certaine opacité: n'étant plus transparente, la représentation est incapable de se poser en «leurre» de la «juste et vraie réalité» (p. 177). Cette opacité de la représentation semble elle-même être fonction d'un régime particulier de l'œuvre. Par exemple, avec la peinture Mère et fille 
(Mother and Child, 1905) de Mary Cassatt, le regard du spectateur «est laissé en retrait et ne peut éprouver qu'à distance l'espace quasi fermé sur lui-même d'une relation qui, faisant boucle, exhibe les limites de la représentation» (p. 177). Le spectateur est ainsi convié à une relation paradoxalement informée par une dynamique de mise à l'écart et de distanciation. Cette expérience est susceptible de se vivre comme un bouleversement, une transformation radicale. C'est le cas avec l'œuvre de Dominique Blain, Traces (1991): "à cette vue, le corps regardant se trouve éprouvé, car faisant l'effort de saisir l'image, il se trouve, du même souffle, compromis" (p. 177). Ainsi, les limites de la représentation sont susceptibles, selon Saint-Gelais, de «modifier notre rapport au monde» (p. 178), voire de nous amener "vers une compréhension différente du sensible dans nos rapports avec soi et autrui » (p. 183).

De tous les textes de ce collectif, celui de Nicolas Tremblay est le seul à aborder le concept d'image à partir du point de vue de l'analyse littéraire, et il le fait avec brio. Il est également l'un des rares à examiner avec autant de rigueur les manières par lesquelles un processus de genèse peut mener à l'individuation d'une représentation objectivée: peinture, photographie, sculpture ou, dans le cas qui nous occupe, œuvre romanesque. S'appuyant sur la stylistique littéraire unique de Réjean Ducharme - et particulièrement sur son roman Gros mots -, Tremblay propose une «herméneutique du sujet créateur»(p. 190). Il s'agit d'enquêter sur la genèse progressive du texte littéraire à partir d'un effort d'abstraction (au sens d'extraction) du flux de pensées prélinguistiques et préconceptuelles en une expression individuée dans l'écriture alphabétique du livre complété ${ }^{16}$. Le récit et les manières particulières de Ducharme mettent en effet en scène, dans le roman Gros mots, ce que Tremblay identifie comme "un simulacre de manuscrit» (p. 190). Ce manuscrit, comme étape intermédiaire entre l'inexprimable de la pensée vivante de l'auteur et la lisibilité objective des lettres alphabétiques, avec ses traces et ses ratures, se laisse voir "comme on regarde une image ou son reflet dans la glace" (p. 197). Il est possible alors de faire l'expérience de "ce qu'on ne saurait et ne devrait voir, l'image des débuts d'avant notre séparation, ne 
reconnaissant plus la distance qu'instaure le signe avec son objet signifié et son Auteur»(p. 197). Nicolas Tremblay suggère en définitive que l'abolition de cette distance recèle un danger, similaire à celui que constitue notre "médiacratie journalistique» (p. 198) : celui consistant à prendre le plus concret pour le plus abstrait, et inversement.

Le dernier texte du collectif constitue une nouvelle tentative de penser les spécificités de l'intermédialité à partir, cette fois, de la double pratique, littéraire et cinématographique, d'Alain Robbe-Grillet. Dans la relation qu'entretiennent images cinématographiques et images littéraires, Stéphanie Veillet situe un espace de création «interelationnel, voire intermédial» (p. 200). Comme dans l'analyse offerte par Emmanuelle Dyotte, la notion d'intermédialité renvoie, à partir des travaux d'Éric Méchoulan, à un terme autologique "qui suppose le dédoublement d'un mouvement consistant à démontrer le mouvement médiatique qui se dédouble lui-même sans arrêt»(p. 201). Cet espace est donc "stimulé par une intermédialité qui constitue en fait le point de rencontre de plusieurs médias dans l'immédiateté de la création» (p. 203). Le régime de représentation de l'image y développe un rythme propre, cadencé par la fragmentation ou la discontinuation fondatrice d'un tel médium. Le musicologue Michel Fano, qui a travaillé sur plusieurs des films de Robbe-Grillet, compose, par exemple, des partitions musicales en faisant se rencontrer des unités sonores produites par la décomposition d'autres sons. Veillet a également recours à la notion barthésienne de "bruissement", suggérant un "bruit limite» multipliant le potentiel d'expression de l'image (p. 208). Le spectateur ou l'" actant du processus intermédial " s'immisce dans les interstices d'un récit fragmenté: son expérience est un processus de liaison, seule unité de laquelle inférer l'unité de la représentation (mouvement qu'adopte d'ailleurs l'auteure du texte, elle-même hantée par la voix de Robbe-Grillet).

Les hommes qui, il n'y a pas si longtemps, circulaient dans les paysages, font aujourd'hui l'expérience des images qui circulent 
entre eux. Admettons un instant que les idées soient également des images dont le régime d'expression serait tout autant sujet à l'analyse que celui des images analysées par les auteurs de ce collectif. Imaginons, par ailleurs, la multiplicité sans cesse changeante des lecteurs de La circulation des images, tous disposés à produire une série indéfinie de lectures analytiques des textes qui y sont présentés. Il faut alors envisager, dans une perspective similaire à celle dont témoignent les thèses marquantes de ce collectif, que ces textes ne représentent pas passivement les objets qu'ils analysent. Ils offrent plutôt l'expression d'une présence créative qui n'acquiert son mouvement et son volume que dans le faisceau complexe des relations qui font se rencontrer les textes - avec leurs objets d'analyse, les théories qu'ils mobilisent, etc. - , leurs auteurs et les lecteurs qui voudront s'y attarder. Aussi serait-il approprié, il me semble, de supposer que la valeur de cet ouvrage est en partie dépendante de la qualité des rapports que ses propositions seront susceptibles de mobiliser. Une remarque qui peut sans doute être généralisée à la circulation d'autres images, d'autres représentations.

\section{Philippe Théophanidis}

Université de Montréal

\section{NOTES}

1. L'ouvrage se compose en partie des textes de quelques-unes des conférences prononcées dans le cadre du colloque international L'image: espace relationnel, tenu à l'Université du Québec à Montréal en février 2004 à l'initiative du groupe de recherche "Chris Marker et la médiation des images" ainsi que de celui "L'intermédialité de l'expérience" du Département d'études littéraires de l'Université du Québec à Montréal. À ces textes viennent également s'ajouter les essais d'autres auteurs, pareillement interpellés par la problématique des rapports à l'image.

2. Le concept d' «image» n'est pas défini dans l'avant-propos qu'offrent les trois responsables de cet ouvrage.

3. Les responsables précisent bien, dans l'avant-propos, que les études rassemblées dans ce collectif "proposent une réactualisation des notions d'intermédialité, de communication et de diffusion» (p. 7). Notons, par ailleurs, que huit des quatorze participants à cet ouvrage sont membres du Centre de recherche sur l'intermédialité fondé, en 1997, à l'Université de Montréal.

4. «[...] cette suppression hâtive de toutes les distances n'apporte aucune proximité : car la proximité ne consiste pas dans le peu de distance" (Heidegger 1958, p. 194). Dans son article, André Habib remarque avec justesse à quel point les ruines "touristiques" ou représentées par les médias font obstacle à "une expérience de la 
médiation du temps " en n'offrant qu'un accès à un "présent perpétuel " faisant l'économie d'une certaine historicité (p. 93-94).

5. Un «hyperréel» sans doute adéquat au régime d'un temps "hypermoderne» marqué économiquement par un "hypercapitalisme» d' "hyperconsommation" coextensif d'une "hyperpolitique» devenue incontrôlable.

6. Voir, par exemple, l'essai fondateur «A World of Pure Experience» dans lequel James (1996, p. 57) affirme: "Knowledge of sensible realities thus come to life inside the tissue of experience. It is made; and made by relations that unroll themselves in time."

7. L'œuvre de Beecroft pose d'emblée un problème d'appréciation pour qui n'a pas eu l'occasion d'assister en direct à ses performances. En effet, le fonctionnement si particulier qu'analyse Dyotte est en partie empêché par sa représentation en images photographiques (telles que produites aux pages 89 à 92, ou encore sur le site officiel de l'artiste).

8. Sur cette question, André Habib se réfere explicitement à la conception de l'écriture allégorique qu'expose Walter Benjamin dans son Origine du drame baroque allemand. Michel Maffesoli évoque dans son texte, d'une manière plus théâtrale, "le fantasme de l'Un" qu'entretiendrait une «intelligentsia moderne» (p. 129-130). Voir également ce qu'en disent Theodor Adorno et Max Horkheimer (1974) dans "Le concept d'Aufklärung" ou encore l'analyse qu'offre Maurice Blanchot (1969) de la forme fragmentaire de l'écriture nietzschéenne dans L'entretien infini.

9. Ce que d'autres auteurs du collectif ont pu appeler ailleurs «autoréférence» ou " autoréflexion".

10. C'est par ailleurs cette perspective qui permet à Dyotte de dégager le potentiel "critique» des performances de Vanessa Beecroft.

11. Toutefois, si cette démarche est effective dans l'espace «intertextuel » qui s'offre à un "regard théorique», quel impact peut-elle avoir sur un regard non informé, voire inattentif? Un tel spectateur serait-il susceptible, comme le suggère Legault, d'être acculé à sa propre "structure voyeuriste" par la mise en scène (p. 122) ? Il est permis d'en douter.

12. Écriture "cinéante", écriture expériencielle et écriture filmique: ce sont là des expressions qui semblent être substituables l'une à l'autre dans l'article de Lucie Roy.

13. Roy écrit exactement: «La modulation a tout à voir avec la perception, celle qui prévaut entre les personnages, celle que l'énonciateur du film leur impose, de même que celle que le spectateur en a ultimement» (p. 158). La chronologie impliquée par ce passage de l'analyse semble contredire l'argument général soutenant la thèse d'une coproduction de l'œuvre et de l'expérience cinématographique.

14. Il faut noter que l'auteure envisage elle-même que son texte puisse être reçu comme "une première étape » dans une analyse en développement (p. 155).

15. Sur le fondement de ce schème "hylémorphique», voir notamment le premier chapitre de L'individu et sa genèse physico-biologique (Simondon 1964).

16. Récit de genèse qui n'est pas sans rappeler ceux qu'évoquent, chacun à leur manière, Charles S. Peirce (1992) et Gilles Deleuze ("Leibniz", cours du 10 mars 1987, disponible en ligne à l'adresse suivante: < http://www.webdeleuze.com/pdf/ fr/Leibniz/100387.zip >) lorsqu'ils tentent d'expliquer comment les termes et les relations peuvent surgir d'un flux chaotique continu. 


\section{RÉFÉRENCES BIBLIOGRAPHIQUES}

Adorno et Horkheimer 1974: Theodor Adorno et Max Horkheimer, "Le concept d'Aufklärung", La dialectique de la raison [1947], Paris, Gallimard, coll. "Tel », 1974, p. 21-57.

Blanchot 1969: Maurice Blanchot, L'entretien infini, Paris, Gallimard, 1969.

Foucault 1966: Michel Foucault, Les mots et les choses, Paris, Gallimard, 1966.

Heidegger 1971 : Martin Heidegger, Essais et conférences [1954], Paris, Gallimard, 1971.

James 1996: William James, "A World of Pure Experience» [1904], Essays in Radical Empiricism, Nebraska, University of Nebraska Press, 1996, p. 39-91.

Peirce 1992: Charles S. Peirce, "A Guess at the Riddle» [1890], The Essential Peirce: Selected Philosophical Writings, vol. 1, Peirce Edition Project (dir.), Bloomington, University of Indiana Press, 1992, p. 245-279.

Simondon 1964: Gilbert Simondon, Lindividu et sa genèse physico-biologique [1964], Paris, Jérôme Millon, 1995. 